\title{
CORRECTING GOES-R MAGNETOMETER DATA FOR STRAY FIELDS
}

\author{
Delano Carter $^{(1)}$, Douglas Freesland ${ }^{(2)}$, Sivakumara K. Tadikonda $^{(3)}$, \\ Jeffrey Kronenwetter ${ }^{(4)}$, Monica Todirita ${ }^{(5)}$, Melissa Dahya ${ }^{(6)}$, Donald Chu ${ }^{(7)}$ \\ (1) Thearality, NASA GSFC Code 417.0, Greenbelt, MD, USA, 20771, delano.r.carter@nasa.gov \\ (2) ACS Engineering, NASA GSFC Code 417.0, Greenbelt, MD, USA, 20771, douglas.c.freesland@nasa.gov \\ (3) Constellation Software Engineering, NASA GSFC Code 417.0, Greenbelt, MD, USA, 20771, \\ sivakumara.k.tadikonda@nasa.gov \\ (4) Chesapeake Aerospace, NASA GSFC Code 417.0, Greenbelt, MD, USA, 20771, jeffrey.a.kronenwetter@nasa.gov \\ (5) NOAA, NASA GSFC Code 417.0, Greenbelt, MD, USA, 20771, monica.todirita-1@nasa.gov \\ (6) ASRC Federal, NASA GSFC Code 417.0, Greenbelt, MD, 20771, melissa.dahya@noaa.gov \\ (7) Chesapeake Aerospace, NASA GSFC Code 417.0, Greenbelt, MD, USA, 20771, donald.chu-1@nasa.gov
}

\begin{abstract}
Time-varying spacecraft magnetic fields or stray fields are a problem for magnetometer systems. While constant fields can be removed with zero offset calibration, stray fields are difficult to distinguish from ambient field variations. Putting two magnetometers on a long boom and solving for both the ambient and stray fields can be a good idea, but this gradiometer solution is even more susceptible to noise than a single magnetometer. Unless the stray fields are larger than the magnetometer noise, simply averaging the two measurements is a more accurate approach. If averaging is used, it may be worthwhile to explicitly estimate and remove stray fields. Models and estimation algorithms are provided for solar array, arcjet and reaction wheel fields.
\end{abstract}

\section{INTRODUCTION}

There are several sources of magnetometer error including noise, calibrations and time-varying spacecraft (stray) fields. In this paper, we consider how to remove stray fields. The plan for GOES-R has been to use its two magnetometers on a long boom first to reduce the effect of stray fields and second to estimate and remove them using a gradiometer algorithm [1]. Our simulations, however, have shown that for the assumed stray fields and noise, simple averaging gives better accuracy.

The first part of this paper provides covariance predictions to explain that observation and to determine when the gradiometer would be preferable to averaging. Averaging does not remove stray fields, and if it is indeed better for GOES-R, then removing those stray fields may be a worthwhile endeavor. The second part of the paper provides models for the solar array, arcjet and reaction wheel fields, and the third describes testing and algorithms for characterizing and removing them.

\section{GRADIOMETRY OR AVERAGING}

Our goal is to measure the ambient field $\vec{B}^{A}$, but our inboard $\vec{B}^{I B}$ and outboard $\vec{B}^{O B}$ magnetometer measurements are corrupted by stray fields $\vec{B}^{S}$

$$
\begin{aligned}
\vec{B}^{I B} & =\vec{B}^{A}+\vec{B}^{S}\left(\vec{r}^{I B}\right) \\
\vec{B}^{O B} & =\vec{B}^{A}+\vec{B}^{S}\left(\vec{r}^{O B}\right)
\end{aligned}
$$

The question is whether it is better to solve for the stray field and correct the ambient field estimate as the gradiometer does or just to average and so reduce noise. Intuitively, we expect that for large stray fields, the gradiometer will be better and that for large noise averaging will prevail. To determine the transition point, we estimate the ambient field error for the two algorithms.

At the magnetometers, the stray field may be approximated by that of a magnetic dipole $\vec{m}$. If the dipole-to-magnetometer vector is $\vec{r}$ with magnitude $r$ and unit vector $\hat{r}$, the stray field is

$$
\vec{B}^{S}=\frac{\mu_{0}}{4 \pi r^{3}}\left(3 \hat{r} \hat{r}^{T}-I_{3}\right) \vec{m}=\beta(\vec{r}) \vec{m}
$$

We will use $\beta$ as shorthand for the coefficient matrix.

\subsection{Gradiometer}

From the dipole equation, one can see that field strength falls off as the third power of distance and that field direction does not change with distance. This led to the following observation model for a pair of magnetometers (inboard IB and outboard OB) so far from the spacecraft that the magnetometers and dipole were effectively collinear.

$$
\vec{y}=\left(\begin{array}{c}
\vec{B}^{I B} \\
\vec{B}^{O B}
\end{array}\right)=\left(\begin{array}{cc}
I_{3} & I_{3} \\
I_{3} & I_{3} / \rho^{3}
\end{array}\right)\left(\begin{array}{l}
\vec{B}^{A} \\
\vec{B}^{S}
\end{array}\right)=H \vec{x}
$$

In this expression, $\rho$ is the ratio of the outboard-toinboard magnetometer distances

$$
\rho=r_{O B} / r_{I B}>1
$$


and $\vec{B}$ is the stray field at the inboard magnetometer. For later use with the averaging covariance, we partition the Jacobian matrix $H$ into ambient and stray field parts $H_{A}$ and $H_{S}$

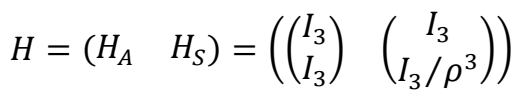

If $\sigma^{2}$ is the observation variance, the minimum variance weighting matrix $W$ is

$$
W=I_{6} / \sigma^{2}
$$

The least squares solution is

or

$$
\vec{x}=\left(H^{T} W H\right)^{-1} H^{T} W \vec{y}
$$

$$
\left(\begin{array}{l}
\vec{B}^{A} \\
\vec{B}^{S}
\end{array}\right)=\frac{1}{\rho^{3}-1}\left(\begin{array}{cc}
-\left(\rho^{3}-1\right) & \rho^{3} \\
\rho^{3} & -\rho^{3}
\end{array}\right)\left(\begin{array}{l}
\vec{B}^{I B} \\
\vec{B}^{O B}
\end{array}\right)
$$

The normal matrix $H^{T} W H$ is

$$
H^{T} W H=\frac{1}{\sigma^{2}}\left(\begin{array}{cc}
2 I_{3} & \left(1+\rho^{-3}\right) I_{3} \\
\left(1+\rho^{-3}\right) I_{3} & \left(1+\rho^{-6}\right) I_{3}
\end{array}\right)
$$

and its inverse is the state covariance matrix $P$

$$
P=\frac{\sigma^{2}}{\left(\rho^{3}-1\right)^{2}}\left(\begin{array}{cc}
\left(1+\rho^{6}\right) I_{3} & -\rho^{3}\left(1+\rho^{3}\right) I_{3} \\
-\rho^{3}\left(1+\rho^{3}\right) I_{3} & 2 \rho^{6} I_{3}
\end{array}\right)
$$

This gives the gradiometer ambient field variance $\sigma_{G}^{2}$ as the upper left corner entry

$$
\sigma_{G}^{2}=\frac{1+\rho^{6}}{\left(\rho^{3}-1\right)^{2}} \sigma^{2}
$$

\subsection{Averaging}

Now, we do the same for the averaging algorithm. If we solve for $\vec{B}^{A}$ as the average of the two magnetometer measurements, the observation model is

$$
\vec{y}=\left(\begin{array}{c}
\vec{B}^{I B} \\
\vec{B}^{O B}
\end{array}\right)=\left(\begin{array}{l}
I_{3} \\
I_{3}
\end{array}\right) \vec{B}^{A}=H_{A} \vec{x}
$$

and $\vec{B}^{A}$ is

$$
\vec{B}^{A}=\frac{1}{2}\left(\begin{array}{ll}
I_{3} & I_{3}
\end{array}\right)\left(\begin{array}{c}
\vec{B}^{I B} \\
\vec{B}^{O B}
\end{array}\right)
$$

Thus, the noise portion $P_{n}$ of the ambient field covariance is

$$
P_{n}=\frac{\sigma^{2}}{2} I_{3}=\left(H_{A}^{T} W H_{A}\right)^{-1}
$$

This is not the total covariance because it ignores the error due to the stray field. To account for this, we use "consider covariance" $P_{c}$ [2]. If $P_{S}$ is the covariance of the stray field itself

$$
P_{S}=\sigma_{S}^{2} I_{3}
$$

its contribution to the ambient field estimate is

$$
P_{c}=T P_{S} T^{T}=\left(P_{n} H_{A}^{T} W H_{S}\right) P_{S}\left(P_{n} H_{A}^{T} W H_{S}\right)^{T}
$$

where the transformation matrix $T$ is

$$
T=\frac{\sigma^{2}}{2} I_{3}\left(\begin{array}{ll}
I_{3} & I_{3}
\end{array}\right) \frac{I_{3}}{\sigma^{2}}\left(\begin{array}{c}
I_{3} \\
I_{3} / \rho^{3}
\end{array}\right)=\frac{\rho^{3}+1}{2 \rho^{3}} I_{3}
$$

The total averaging ambient field covariance $P_{A}$ is the sum of $P_{n}$ and $P_{c}$

$$
P=P_{n}+P_{c}
$$

The total variance of the ambient field solution error $\sigma_{A}^{2}$ is

$$
\sigma_{A}^{2}=\frac{\sigma^{2}}{2}+\left(\frac{\rho^{3}+1}{2 \rho^{3}}\right)^{2} \sigma_{S}^{2}
$$

\subsection{Algorithm Comparison}

If the gradiometer variance $\sigma_{G}^{2}$ is greater than the averaging variance $\sigma_{A}^{2}$, averaging is the better choice. The ratio of averaging to gradiometer standard deviations is

$$
\frac{\sigma_{A}}{\sigma_{G}}=\sqrt{\frac{\left(\rho^{3}-1\right)^{2}}{1+\rho^{6}}\left(\frac{1}{2}+\left(\frac{\rho^{3}+1}{2 \rho^{3}}\right)^{2} \frac{\sigma_{S}^{2}}{\sigma^{2}}\right)}
$$

Figure 1 shows this ratio for different values of stray field-to-noise and inboard-to-outboard distance $\rho$

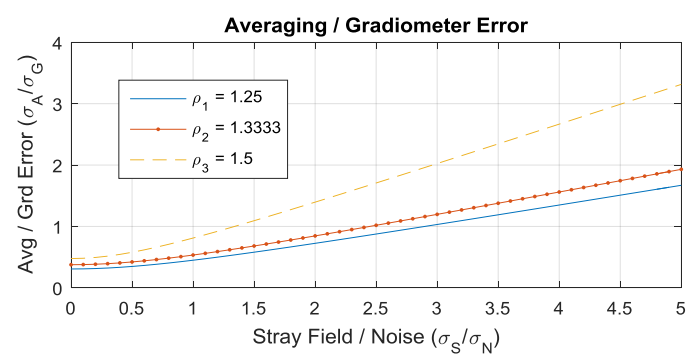

Figure 1. Averaging / Gradiometer Error

Equating the two variances gives an equation for the stray field variance beyond which the gradiometer becomes the better choice

$$
\frac{1+\rho^{6}}{\left(\rho^{3}-1\right)^{2}} \sigma^{2}=\frac{\sigma^{2}}{2}+\left(\frac{\rho^{3}+1}{2 \rho^{3}}\right)^{2} \sigma_{S}^{2}
$$

Solving for the transition ratio gives

$$
\frac{\sigma_{S}}{\sigma}=\sqrt{2} \frac{\rho^{3}}{\rho^{3}-1}
$$

This expression suggests that for the assumed outboardto-inboard distance ratio of 1.33 , the stray field variance would have had to have been at least twice that of the 
noise for the gradiometer to be preferable to averaging. In our simulations, we assumed that noise and stray field standard deviations were both $0.1 \mathrm{nT}$.

\section{STRAY FIELD MODELS}

Because we believe that we can calibrate for magnetometer zero offsets and static spacecraft fields, our primary concern now is with time-varying, i.e. stray fields [3]. Every spacecraft assembly was measured for compliance with the magnetic specs, and the three that were found to generate the largest stray fields were the solar array, the arcjet thrusters and the reaction wheels.

\subsection{Solar Array Model}

The solar array is divided into circuits, and each may be modeled as a dipole. To reduce the solar array field, half of those circuits were wound clockwise and the other half counterclockwise. Because of the distance separating the circuits and the magnetometers, we approximate the solar array field with that of the net dipole placed at the centroid of the array as shown in Figure 2.

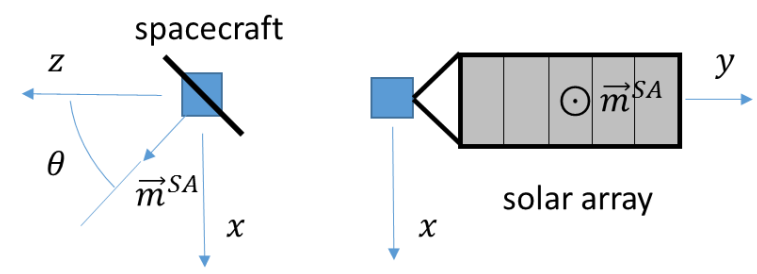

Figure 2. Solar Array Drive Angle $\theta$

The solar array dipole moment vector $\vec{m}^{S A}$ is a function of the effective solar array circuit area $A^{S A_{i}}$, current $i^{S A_{i}}$ and the solar array drive angle $\theta$ as shown in Figure 1

$$
\vec{m}^{S A}=\sum_{i=1}^{n} A^{S A_{i}} i^{S A_{i}}\left(\begin{array}{c}
\sin \theta \\
0 \\
\cos \theta
\end{array}\right)
$$

The solar array field is then

$$
\vec{B}^{S A}=\beta\left(\begin{array}{c}
\sin \theta \\
0 \\
\cos \theta
\end{array}\right)\left(\sum_{i=1}^{n} A^{S A_{i}} i^{S A_{i}}\right)
$$

\subsection{Arcjet Model}

For inclination control, there are four arcjets on the $-y$ (north) face of the spacecraft. They are fired two at a time and do not themselves generate much field, but the electrical current they require does. The two arcjet-pair dipole moments $\vec{m}^{a / b}$ are a function of the current $i^{a / b}$ and the circuit areas projected onto the yz (x-component), zx (y-component) and xy (z-component) planes as shown in Figure 3.

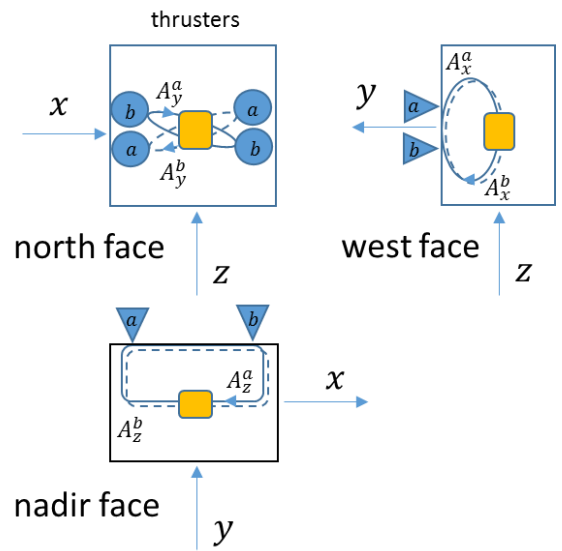

Figure 3. Arcjet Dipole Moment Areas $A^{N / S}$

These areas may be written as a vector $\vec{A}^{a / b}$ such that the two arcjet dipoles take the form

$$
\vec{m}^{a / b}=\left(\begin{array}{lll}
A_{x}^{a / b} & A_{y}^{a / b} & A_{z}^{a / b}
\end{array}\right)^{T} i^{a / b}=\vec{A}^{a / b} i^{a / b}
$$

The arcjet fields can then be written as a linear function of the arcjet current

$$
\vec{B}^{a / b}=\beta \vec{A}^{a / b} i^{a / b}
$$

\subsection{Reaction Wheel Model}

Reaction wheel residual magnetization produces a dipole moment that varies sinusoidally at the rotation rate. If the rotation rate $\omega^{R W_{i}}$ is within magnetometer passband, the magnetometer sees it. The moment vector is in the rotor plane and may be expressed in terms of unit basis vectors $\hat{u}$ and $\hat{v}$ fixed in the reaction wheel assembly frame as shown in Figure 4.

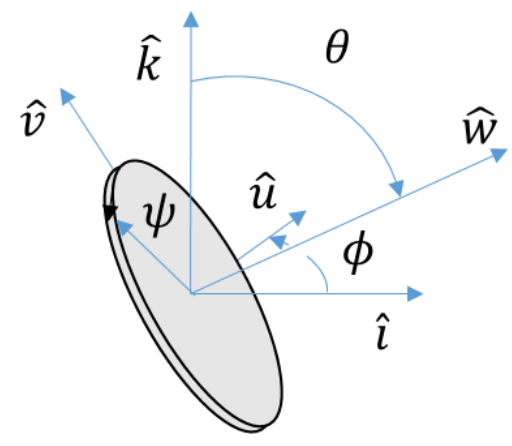

Figure 4. Reaction Wheel Phase Angle $\psi$

Predicting reaction wheel fields requires knowing the magnitude of the rotor dipole moment $m^{R W_{i}}$, the wheel speeds $\omega^{R W_{i}}$ and the rotor phase $\psi^{R W_{i}}$. If we neglect the distance between the wheels, we can sum the moments as

$$
\vec{m}^{R W}=\sum_{i=1}^{n} m^{R W_{i}}\left(\cos \psi^{R W_{i}} \hat{u}^{R W_{i}}+\sin \psi^{R W_{i}} \hat{v}^{R W_{i}}\right)
$$

where the $\hat{u}_{i}$ and $\hat{v}_{i}$ are 


$$
\begin{gathered}
\hat{u}_{i}=\hat{k} \times \widehat{w}_{i} /\left|\hat{k} \times \widehat{w}_{i}\right| \\
\hat{v}_{i}=\widehat{w}_{i} \times \hat{u}_{i} /\left|\widehat{w}_{i} \times \hat{u}_{i}\right|
\end{gathered}
$$

The plan then would be to compute these dipole moment vectors, calculate the fields they produce at the magnetometers and subtract them from the inboard and outboard readings before averaging

$$
\vec{B}^{R W}=\beta \vec{m}^{R W}
$$

\section{STRAY FIELD CHARACTERIZATION}

To remove the solar array, arcjet and reaction wheel fields as outlined above, we have to know:

1. Solar array drive angle, circuit areas and currents

2. Arcjet sensitivities and currents

3. Rotor in-plane dipole moments, rotation rates and phases

\subsection{Solar Array Characterization}

The solar array drive angle and currents come in telemetry, so the important remaining items to determine are the circuit areas. On the ground, known currents were forced through the solar array circuits, and the resulting magnetic fields were measured. These measurements were turned into dipole moments again for the observation model

$$
\left(\begin{array}{c}
\vec{B}^{1 m_{i}} \\
\vec{B}^{2 m_{i}}
\end{array}\right)=\left(\begin{array}{c}
\beta\left(\vec{r}^{1 m}\right) \\
\beta\left(\vec{r}^{2 m}\right)
\end{array}\right) \vec{m}_{i}^{S A_{i}}
$$

These were then normalized by the applied currents to give the effective areas $A^{S A_{i}}$ for each circuit

$$
A^{S A_{i}}=m^{S A_{i}} / i^{S A_{i}}
$$

On-orbit we plan to dither the solar array and estimate its dipole moment from the resulting field variation. We have to choose dither amplitude and frequency that satisfy the solar array angular velocity $\omega$ and acceleration $\alpha$ constraints while changing the field appreciably at a frequency well above that of ambient variations.

Assuming sinusoidal dither with amplitude $\Theta$ and angular velocity $\Omega$, the solar array drive angle $\theta$ is equal to

$$
\theta=\Theta \sin \Omega t
$$

Limits on the maximum solar array angular velocity $\omega$ and acceleration $\alpha$ constrain $\Omega$ and $\Theta$ are

$$
\begin{aligned}
& \omega=\dot{\theta} \leq \Omega \Theta \leq \omega_{\max } \\
& \alpha=\ddot{\theta} \leq \Omega^{2} \Theta \leq \alpha_{\max }
\end{aligned}
$$

Dithering causes the solar array field to vary about a nonzero mean value. To estimate the dipole moment, we first subtract the average field value from both the observation and the prediction. A direct search then minimizes the sum of squared errors.

With one hour of $5^{\circ}$ dithering, we are able estimate the solar array dipole moment to $0.5 \mathrm{Am}^{2}(1 \sigma)$ accuracy. This is less accurate than the ground measurements but does provide a check under flight-like conditions. As shown in Figure 5, if we were to use the in-flight estimates, we would expect to reduce solar array stray fields by half.
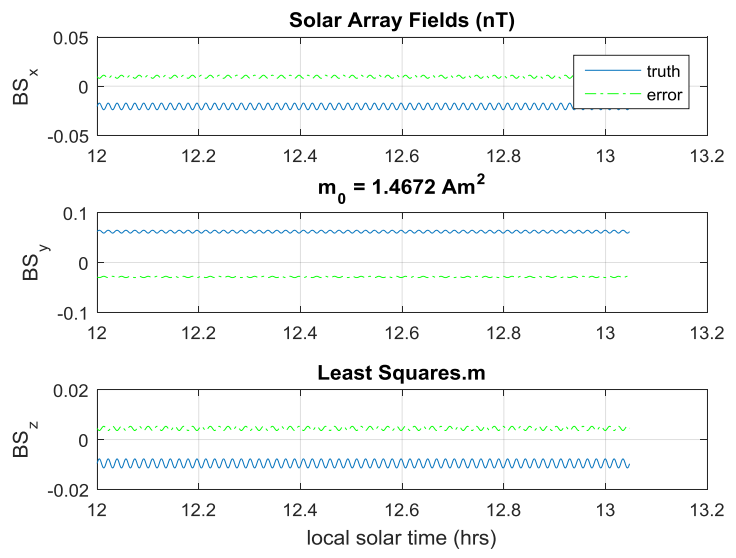

Figure 5. Reduction of Solar Array Fields

\subsection{Arcjet Characterization}

Unfortunately, it is not possible to fire the arcjets on the ground with the cabling in a flight-like configuration, so arcjet magnetic characterization has to be on-orbit. In normal operations, the arcjets are fired every few days in one long pulse. The on- and off-transitions should be quite sharp, and we know when to look for them. So it should be possible to observe them accurately. In addition, we will have telemetry for the currents.

As above, the procedure would be to solve a least squares problem for the arcjet dipole moment using the change in the magnetometer observations $\Delta \vec{B}^{I B / O B}$ before and after the transitions

$$
\left(\begin{array}{c}
\Delta \vec{B}^{I B} \\
\Delta \vec{B}^{O B}
\end{array}\right)=\left(\begin{array}{c}
\beta\left(\vec{r}^{I E}\right) \\
\beta\left(\vec{r}^{O E}\right)
\end{array}\right) \vec{m}^{a / b}
$$

where $\vec{r}^{I E}$ and $\vec{r}^{O E}$ are the arcjet electrical power unit position $\vec{r}^{E}$ to magnetometer position vectors

$$
\begin{gathered}
\vec{r}^{I E}=\vec{r}^{I B}-\vec{r}^{E} \\
\vec{r}^{O E}=\vec{r}^{O B}-\vec{r}^{E}
\end{gathered}
$$

Without noise, it would only take one $a$ and one $b$ arcjet pair pulse to determine the fields. The magnetometer 
noise $\sigma$ plus the ambient field variability $\sigma_{B}$ make it necessary to average multiple firings. If over the short time $(0.25 \mathrm{sec})$ it takes the magnetometer to respond to the step transition, the ambient field does not change, the only noise is from the magnetometer itself. In this case, it would take 100 transitions (50 pulses) to reduce the $0.10 \mathrm{nT}$ magnetometer noise to the $0.01 \mathrm{nT}$ level we might want for arcjet field knowledge.

\subsection{Reaction Wheel Characterization}

Before assembly, we measured the magnetic dipole moment of each of the six reaction wheel rotors. Assuming the dipole moment does not change, if we knew the rotor orientations, i.e. phase angles, we could predict the reaction wheel fields at the magnetometers.

Unfortunately, there is no rotor phase telemetry, so we have to estimate the phases. We use tachometer wheel speeds to propagate phase between observations, i.e. times when the rates are within the magnetometer passband. During those times, we estimate the rotor fields and remove them from the magnetometer readings.

Over the course of a day, wheel speeds may look like what is displayed in Figure 6. When wheel speeds are within the magnetometer passband, measurements are corrupted. Wheel speeds are used as inputs to a bank of Least Mean Squares (LMS) based adaptive filters [4, 5]. Because six wheels may be within the magnetometer measurement passband, the required number of parallel LMS adaptive filters is six, i.e. one per wheel.

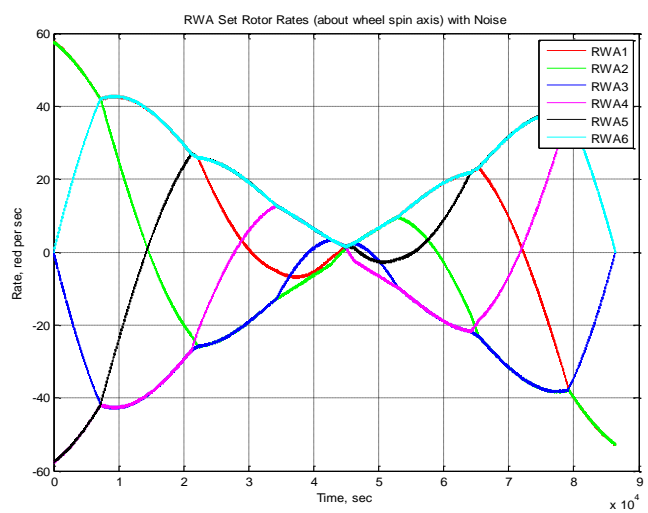

Figure 6. Typical Reaction Wheel Speeds

Each LMS adaptive filter is responsible for estimating the wheel dipole phase angle and amplitude. The estimated magnetic dipole vector is then used to remove the wheel field from the magnetometer outputs. The parallel bank of filters is shown in Figure 7.

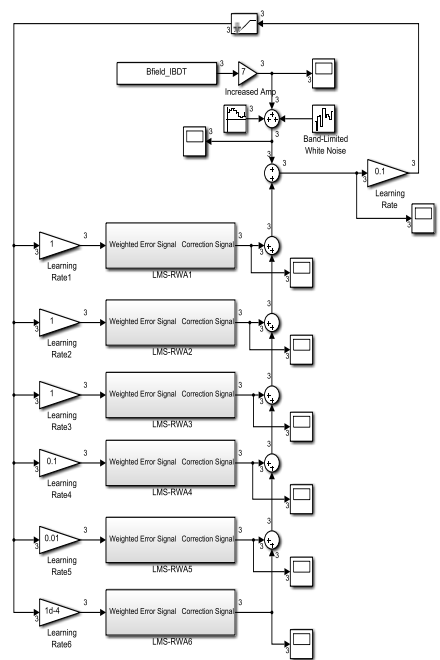

Figure 7. Bank of Parallel LMS Adaptive Filters

There are two inputs to each LMS adaptive filter. One is the tachometer signal, and the other is the filter effectivity error. Filter effectivity error is a measure of how well the filter is removing the undesired wheel field. Based on these two inputs, the LMS adaptive filter generates a correction signal to remove the dipole field.

To prevent the LMS filters from competing with each other in a detrimental manner, the learning rates for the six LMS adaptive filters are skewed. This has the effect of permitting some filters to converge to wheel magnetic dipole signals quicker than other filters. In this manner, filters can self-select which magnetic dipoles to converge to when multiple wheel speeds are within the measurement band. The remaining filters then converge to the remaining wheel magnetic dipole signals.

Figure 8 shows the predicted reduction in reaction wheel speeds using this approach.
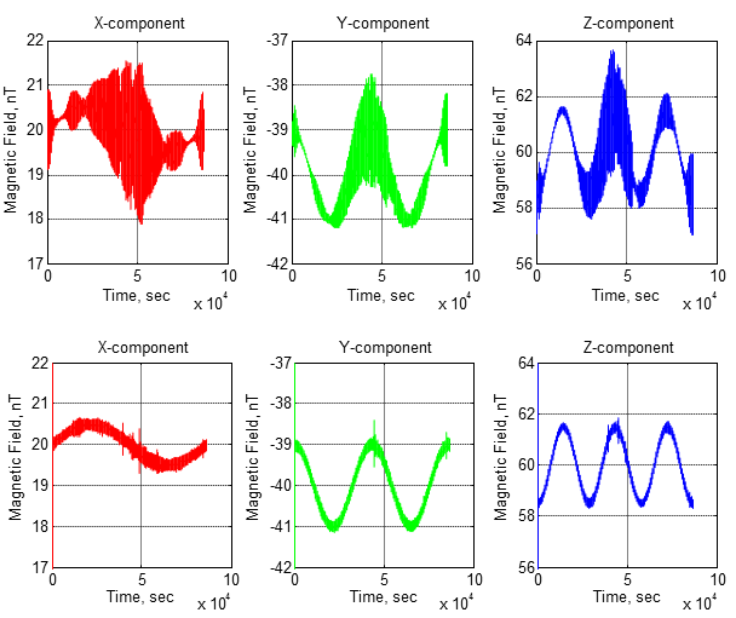

Figure 8. Reduction in Reaction Wheel Fields 
The top row shows the simulated reaction wheel fields superimposed on a sinusoidally-varying ambient field. The traces are wide when the wheel speeds go through the magnetometer passband. The bottom row shows the magnetometer readings after being corrected with the LMS filter estimates.

\section{CONCLUSION}

This paper proposes ways of dealing with time-varying stray magnetic field errors in space-based magnetometer systems. It examines gradiometer noise susceptibility and recommends when to use gradiometry and when to average. Unless stray fields are twice as large as magnetometer noise, covariance analysis suggests that it is preferable to average rather than use the gradiometer.

It also outlines models for three common sources of stray fields, i.e. solar array, arcjets and reaction wheels, and suggests how the necessary parameters can be measured and the stray fields removed. Because averaging does not remove stray fields, if it is chosen over gradiometry, it may be worthwhile to add these steps to ground processing.

One question not covered is on-orbit performance verification. How will we know that any corrections we make actually help? There may be times when we are collocated with other GOES satellites and can compare measurements, but most of the time there will be no reference nearby. This and other operations questions remain to be addressed.

\section{ACKNOWLEDGEMENTS}

The authors are grateful for their support under NASA Contract NNG14CR58C and thank Chris Chastain, Robert Dence, Doug Westbury, Beth Shoemaker and Andrew Grimes of Lockheed Martin Space Systems for their design, development, analysis and accommodation of the GOES-R magnetometer subsystem. Finally, the authors thank intern Elizabeth Singleton for her work on solar array characterization.

\section{REFERENCES}

1. Ness, N.F. et al. (1971). "Use of Two Magnetometers for Magnetic Field Measurements on a Spacecraft", Journal of Geophysical Research, vol. 76, no. 16 , pp. 3564-3573.

2. Bierman, G. (2006). Factorization Methods for Discrete Sequential Estimation, Dover, pp. 164, 166, 171-178.

3. Todirita, M. et al., "Maintaining Gradiometer Accuracy On-Orbit", 2012 ESA Workshop on Aerospace EMC, May, 2012.
4. Elliott, S.J. and P.A. Nelson, "Active Noise Control”, IEEE Signal Processing Magazine, October 1993, pp. 12-35.

5. Hakansson, L., The Filtered-X LMS Algorithm, http://citeseerx.ist.psu.edu/viewdoc/download?doi= 10.1.1.137.3836\&rep=rep1\&type=pdf 